\title{
Muros de gaviones y pilotes para la protección de ducto de gas de Camisea en la provincia de La Convención, en Cusco, Perú
}

\author{
Gaviones walls and piles for the protection of duct of gas of Camisea \\ in the county of La Convención, in Cusco, Peru
}

\author{
Luis Alberto Páucar Montaño*
}

http://dx.doi.org/10.21503/CienciayDesarrollo.2011.v13.04

\section{RESUMEN}

El presente trabajo se realizó para la protección del ducto de gas que corresponde al proyecto GAS DE CAMISEA, ejecutado por Transportadora del Gas del Perú (TGP), que a su vez es representada por Compañía Operadora del Gas del Amazonas (COGA). La construcción la realiza la empresa ítaloargentina TECHINT S.A.

Esta obra, hecha en el departamento de Cusco, provincia de La Convención, distrito de Echarate, en el tramo que va desde desde el KP 0+000 (Malvinas) al KP 180+000, perteneciente al sector ceja de selva.

El presente informe se realizó básicamente para definir el tipo de proceso de remoción en masa desarrollado y las razones del mismo, así como para plantear y diseñar las obras necesarias para asegurar la estabilidad del DDV.

Tuvo como objetivo también describir adecuadamente las obras diseñadas en el presente informe para su ejecución en campo, obras concebidas para proteger la estabilidad del talud y garantizar la seguridad del ducto en el tramo de esta zona del sector selva.

Palabras clave: pilote, ducto, talud, nivel freático, canal colector.

\section{ABSTRACT}

The present work was carried out for the protection of the duct of gas that corresponds to the project GAS DE CAMISEA, executed by Transportadora of the Gas of Peru (TGP) that in turn is represented by Company Operator of the Gas of the Amazons (COGA). The construction carries out it the italoArgentinean company TECHINT S.A.

This work, made in the department of Cusco, county of The Convention, district of Echarate, in the tract that goes from from KP $0+000$ (Malvinas) to KP $180+000$, belonging to the sector forest brow..

The formless present was carried out basically to define the type of developed process of mass removal and the reasons of the same one, as well as to outline and to design the necessary works to assure the stability of DDV.

This investigation also had as objective to describe the works designed report presently for their execution in field appropriately, works conceived to protect the stability of the bank and to guarantee the security of the duct in the tract of this area of the sector forest.

Key words: pile, duct, bank, phreatic level, channel collector.

\footnotetext{
* Ingeniero civil. Licenciado por la Universidad Alas Peruanas. (e-mail: lapm_35@hotmail.com)
} 


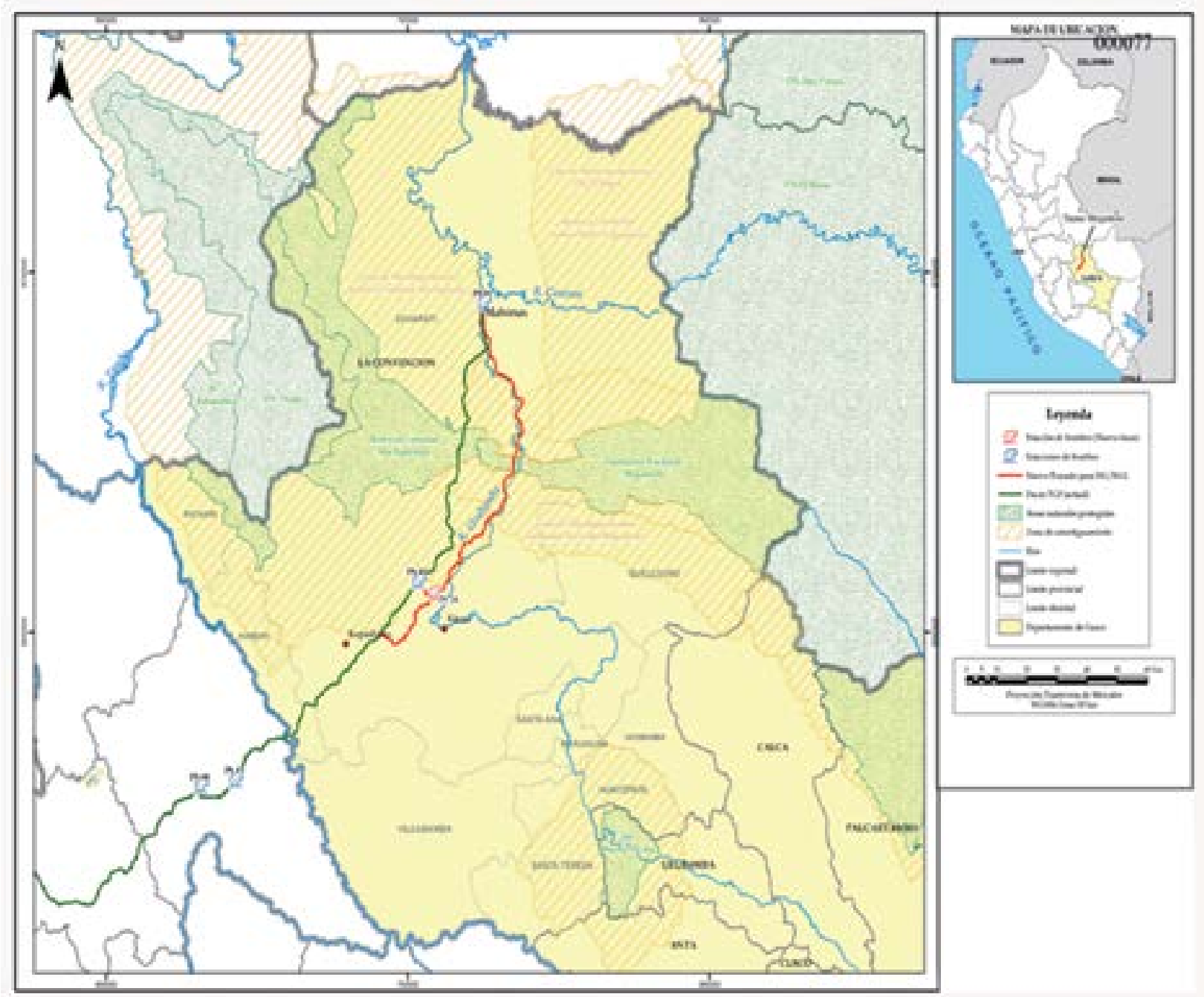

Figura 1. Plano de ubicación.

\section{INTRODUCCIÓN}

En mayo de 2006, luego de identificar un movimiento que involucraba el DDV entre las progresivas K67+300 y KP67+500 y comprobar, mediante las lecturas de Strain Gages, que en el tubo de NGL, a la altura del KP67+500, la manta estaba corrida $200 \mathrm{~mm}$ en la junta J6753, se elaboró el Plan de Trabajo 01 OTGE IT $0160 \mathrm{KP} 067+700$, que tenía como objetivo asegurar la estabilidad de los ductos ante la potencial amenaza que presentaba el escarpe del deslizamiento del acopio lateral contiguo, a la derecha del DDV. Las obras propuestas en dicho informe técnico estaban encaminadas a controlar y estabilizar la masa susceptible de movilizarse y evitar un posible movimiento retrogresivo que afectara a los tubos.

El Plan de Trabajo (PDT) mencionado se desarrolló después de detectar en el costado derecho del DDV el deslizamiento de un acopio lateral que se había dispuesto sobre una ladera de pronunciada pendiente. Las dimensiones del movimiento en ese momento eran de $15 \mathrm{~m}$ de largo y $30 \mathrm{~m}$ de ancho, con un escarpe principal de $1,5 \mathrm{~m}$ de altura. En la base del movimiento se observaba la roca, conformada por una arenita cuarzosa con matriz arcillosa de color café. Según el reporte, la presencia de roca en la base de los tubos era un indicativo de que el movimiento no representaba una amenaza alta 
para la estabilidad de los tubos; así mismo, el buzamiento de las capas era favorable para la estabilidad del terreno.

Las obras de remediación mencionadas en el PDT 01 OTGE IT 016 KP $067+700$ estaban compuestas por un muro de gaviones en sacos de cemento de $2 \mathrm{~m}$ de altura por $30 \mathrm{~m}$ de longitud, con filtros en su trasdós, perfilado y con reconformación del terreno en la zona del escarpe e implementación de trinchoterrazas estabilizadoras.

Durante la temporada de inspección del DDV 2008-2009, realizada por los Grupos de Vigilancia Continuos (GVC), se identificó en el informe 009_Insp del KP 67+000 al 71+000_28 1108 , la reaparición del escarpe en la zona. Dicho punto fue visitado, analizado y valorado en la matriz de riesgo geotécnico, tras de lo cual se definió que era de nivel IV.

\section{Descripción del problema}

En el costado derecho del DDV (sentido de flujo), se observa un escarpe antiguo que dista del ducto NGL $1,5 \mathrm{~m}$ en su sitio más crítico. Este escarpe presenta una reactivación del movimiento detectado en el año 2006.

Las inspecciones realizadas por los GVC en el DDV reportan un incremento en el número de grietas y escarpes con tendencia retrogresiva, asociados con un deslizamiento complejo que involucra superficies de falla rotacionales de gran radio con tendencia traslacional y direcciones de movimientos no homogéneas y producidas en diferentes etapas, así como el hundimiento y desplazamiento del terreno, dentro del cual se encuentra, en la parte baja, el muro de gaviones construido en el año 2006, que presenta deformaciones considerables generadas por asentamientos.
Lazonahasidoobjetodeestudioyobservación constante desde el primer indicio de movimiento, que se detectó en el año 2006. Para esta época se diseñaron obras de remediación enfocadas en la protección del DDV y la tubería, sin pretender detener por completo el movimiento. En el año 2008, y como medida de control, se instalaron piezómetros e inclinómetros en la zona, con el fin de controlar los desplazamientos del terreno y determinar con certeza la profundidad de la superficie de falla.

\section{Características del movimiento}

Sobre el costado derecho del DDV, se localiza un deslizamiento complejo con carácter retrogresivo que comprende todo el acopio lateral entre las progresivas KP67+300 y KP67+500. Durante las últimas inspecciones de los GVC, se observó una reactivación del escarpe.

El tipo de movimiento en el área es complejo, pues presenta superficies de falla rotacionales de gran radio y bloques con movimiento traslacional en la zona de contacto entre la roca y el material blando. Se presenta erosión en la pata del deslizamiento por el paso de un cauce de gran trasporte en la temporada de lluvias, lo cual disminuye el confinamiento de la zona de rellenos en la parte alta. El material de relleno está altamente saturado y presenta grietas de tracción escalonadas, las mismas que llegan hasta el DDV. Todo este movimiento tiene una dirección SW, y las aguas de descole de los cortacorrientes ayudan a saturar el terreno que no se encuentra compacto, provocando un movimiento lento de la masa de suelo. Así mismo, le erosión y el hecho de que los estratos se encuentren muy fracturados ayudan o favorecen el movimiento del terreno.

En la tabla 1 se presentan las principales características del movimiento que afecta al KP 67+500. 
Tabla 1. Características del movimiento en acopio del KP 67+300 a KP 67+500.

\begin{tabular}{|c|c|c|}
\hline Localización & Tipo & Características \\
\hline \multirow{5}{*}{$\begin{array}{c}\text { Costado derecho DDV } \\
\text { KP } 67+300 \text { a KP } 67+500 \\
\text { N: } 8639002 \mathrm{~m} \\
\text { E: } 713526 \mathrm{~m} \\
\text { Elevación: } 1367 \mathrm{msnm}\end{array}$} & \multirow{5}{*}{$\begin{array}{l}\text { Movimiento complejo } \\
\text { con tendencia } \\
\text { retrogresiva }\end{array}$} & Longitud de escarpe $=80 \mathrm{~m}$ \\
\hline & & Largo $\mathrm{L}=100 \mathrm{~m}$ \\
\hline & & Altura del escarpe $\mathrm{h}=1,50 \mathrm{~m}$ \\
\hline & & Ángulo del escarpe $\square=80^{\circ}$ \\
\hline & & $\begin{array}{l}\text { Inclinación del terreno adyacente } \\
\text { al escarpe principal } \square=25^{\circ}-30^{\circ}\end{array}$ \\
\hline
\end{tabular}

Topografía

Con el fin de localizar las obras existentes sobrela zona de estudio, grietas, escarpes, eje delos ductos y cada uno de los elementos que permiten visualizar mejor el fenómeno y modelar el tipo de falla, se realizó el relevamiento topográfico del sector, a partir del cual se elaboraron los perfiles topográficos. En la figura 2 se presenta el relevamiento topográfico del sector.

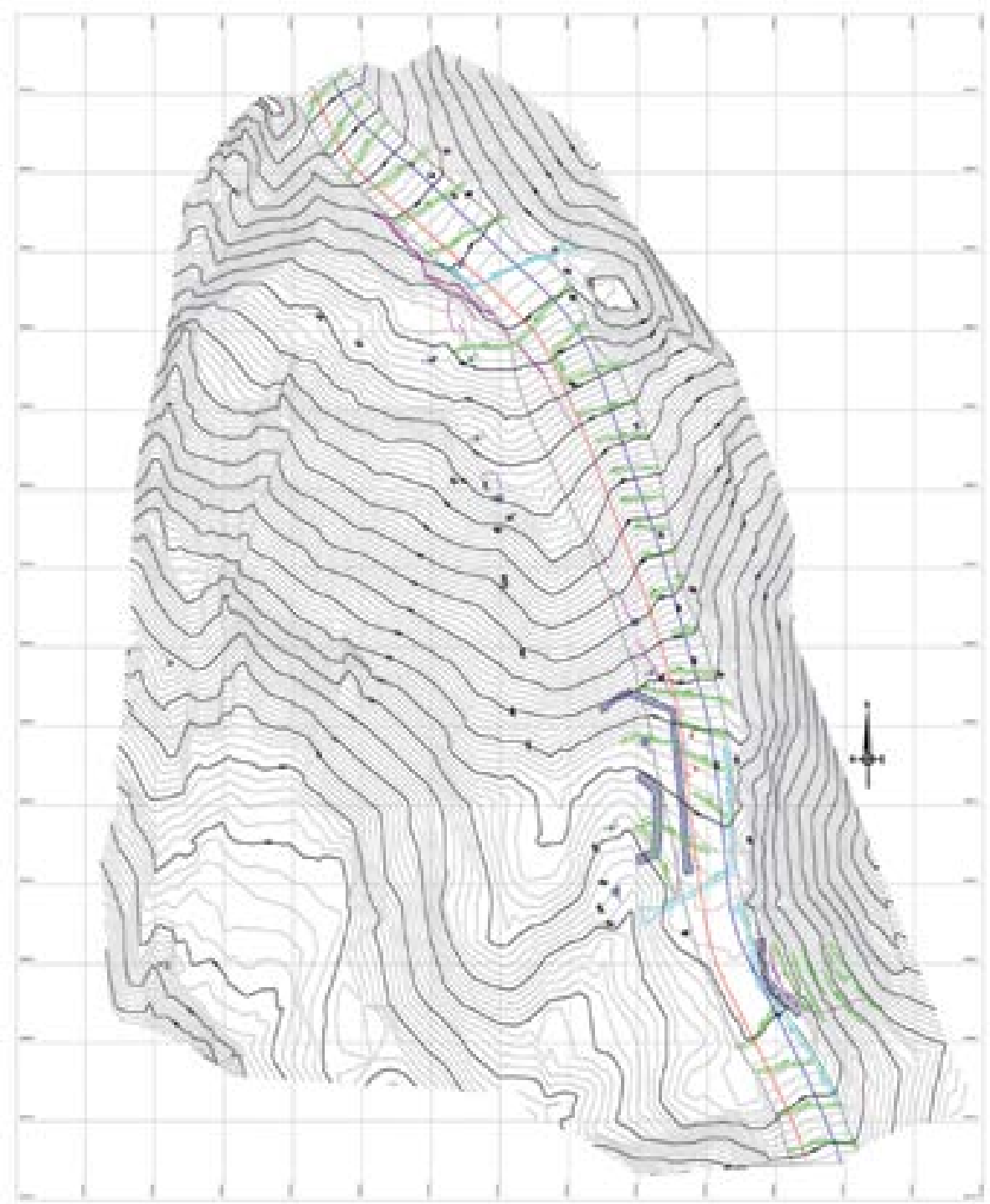

Figura 2. Relevamiento topográfico. 
La progresiva está ubicada en la parte alta de una línea divisoria de aguas (por donde transcurre el DDV), por una ladera de pendientes altas y cerca del nacimiento de un cuerpo de agua. Esta quebrada pertenece a una microcuenca que está delimitada por los ríos Poyentimari y Mantalo, en la parte baja del eje principal de conducción y entrega de la cuenca.

\section{MATERIAL Y MÉTODOS}

\section{Objetivos}

La presente investigación tiene como objetivos:

- Definir el tipo de proceso de remoción en masa que se presenta y sus causas.

- Plantear y diseñar las obras necesarias para asegurar la estabilidad del DDV.
- Describir adecuadamente las obras diseñadas en el presente informe para su ejecución en campo.

Exploración del subsuelo

\section{Sondeos manuales}

En el sector que comprende el fenómeno de remoción en masa del Kp 67+500, se realizaron 68 sondeos manuales con recuperación de muestras alteradas mediante el ensayo de penetración estándar (SPT), ejecutados en 2 etapas. La primera, de 48 sondeos, se desarrolló entre abril y mayo de 2008, y la segunda, de 20 sondeos, en julio de 2009. La localización de los sondeos se determinó en campo con el fin de cubrir la mayor parte del área de trabajo y de facilitar la caracterización del subsuelo. En la figura 3 se presentan la ubicación del lugar donde se realizaron los sondeos.

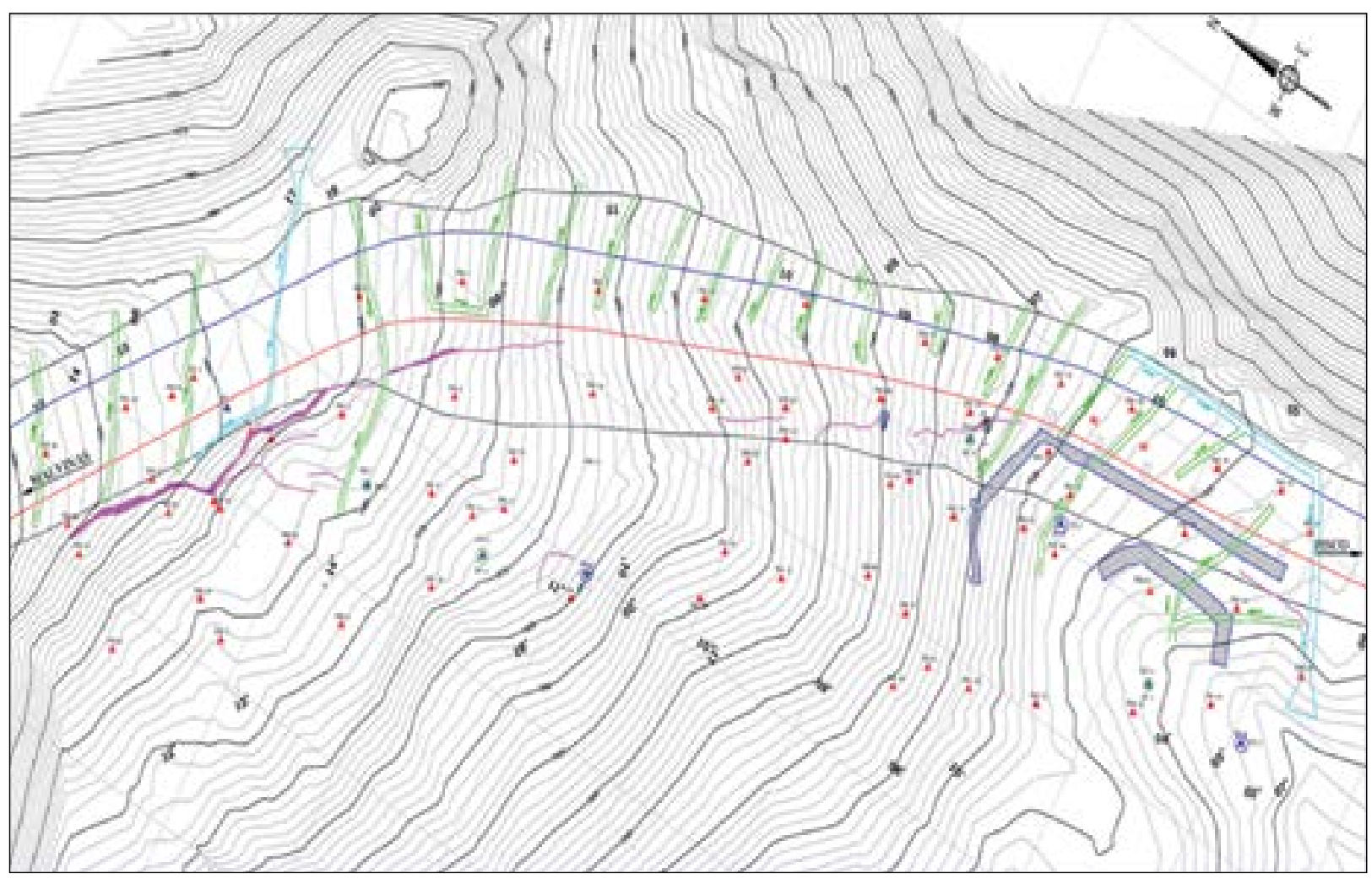

Figura 3. Localización de los sondeos. 


\section{Profundidad de los sondeos manuales}

Dadas las condiciones del equipo manual empleado, la profundidad promedio lograda por este fue de 6,0 m. En la tabla 2 se presentan las profundidades alcanzadas en los sondeos.

Tabla 2. Profundidades de los sondeo.

\begin{tabular}{|c|c|c|c|c|c|c|c|c|c|c|c|c|c|}
\hline SONDEO & $\begin{array}{l}\text { Prof. } \\
\text { (m) }\end{array}$ & SONDEO & $\begin{array}{l}\text { Prof. } \\
\text { (m) }\end{array}$ & SONDEO & $\begin{array}{l}\text { Prof. } \\
\text { (m) }\end{array}$ & SONDEO & $\begin{array}{l}\text { Prof. } \\
(\mathrm{m})\end{array}$ & SONDEO & $\begin{array}{l}\text { Prof. } \\
\text { (m) }\end{array}$ & SONDEO & $\begin{array}{l}\text { Prof. } \\
\text { (m) }\end{array}$ & SONDEO & $\begin{array}{l}\text { Prof. } \\
\text { (m) }\end{array}$ \\
\hline SM 1 & 1,5 & SM 11 & 7.1 & SM 21 & 8 & SM 31 & 3.5 & SM 41 & 7.2 & SM 51 & 7.5 & SM 61 & 7.5 \\
\hline SM 2 & 5,5 & SM 12 & 9,5 & SM 22 & 8 & SM 32 & 5,5 & SM 42 & 8,5 & SM 52 & 5 & SM 62 & 7,5 \\
\hline SM 3 & 7 & SM 13 & 9 & SM 23 & 4 & SM 33 & 7,2 & SM 43 & 7,3 & SM 53 & 1,3 & SM 63 & 5,1 \\
\hline SM 4 & 6,8 & SM 14 & 5,5 & SM 24 & 3,5 & SM 34 & 8,5 & SM 44 & 6 & SM 54 & 2,4 & SM 64 & 7,5 \\
\hline SM 5 & 7,7 & SM 15 & 9 & SM 25 & 4,5 & SM 35 & 5,8 & SM 45 & 2,7 & SM 55 & 4,2 & SM 65 & 7,5 \\
\hline SM 6 & 2,5 & SM 16 & 6,7 & SM 26 & 8 & SM 36 & 7,2 & SM 46 & 1,5 & SM 56 & 5,1 & SM 66 & 7,5 \\
\hline SM 7 & 6,8 & SM 17 & 6 & SM 27 & 6,5 & SM 37 & 8,5 & SM 47 & 6 & SM 57 & 3,1 & SM 67 & 7,5 \\
\hline SM 8 & 9 & SM 18 & 6,5 & SM 28 & 7,4 & SM 38 & 5,5 & SM 48 & 1,5 & SM 58 & 7,2 & SM 68 & 4,5 \\
\hline SM 9 & 7,5 & SM 19 & 8,7 & SM 29 & 5,5 & SM 39 & 3,5 & SM 49 & 2,1 & SM 59 & 7 & & \\
\hline SM 10 & 9,5 & SM 20 & 6,7 & SM 30 & 5 & SM 40 & 5,8 & SM 50 & 2,8 & SM 60 & 7,5 & & \\
\hline
\end{tabular}

Sondeos mecánicos (profundos)

Dado que en 24 de los sondeos manuales realizados no se pudo encontrar el rechazo, con profundidades mayores de $7 \mathrm{~m}$ se decidió ejecutar 8 sondeos profundos por el método de rotación. Dicha etapa se desarrolló en el mes de enero del año 2009.

Tabla 3. Profundidad de Sondeos Profundos.

\begin{tabular}{c|c|c|c|c|c|c|c|c} 
SONDEO & 1 & 2 & 3 & 4 & 5 & 6 & 7 & 8 \\
\hline Profundidad (m) & 19 & 12 & 19 & 19 & 19 & 20 & 17,85 & 10 \\
\hline
\end{tabular}

Perfil promedio del subsuelo

De acuerdo con la información suministrada por los registros de exploración, se determinó el perfil promedio característico para la zona. Este último se muestra en la tabla 4. 


\section{Tabla 4. Perfil promedio del subsuelo en el Kp 67+500.}

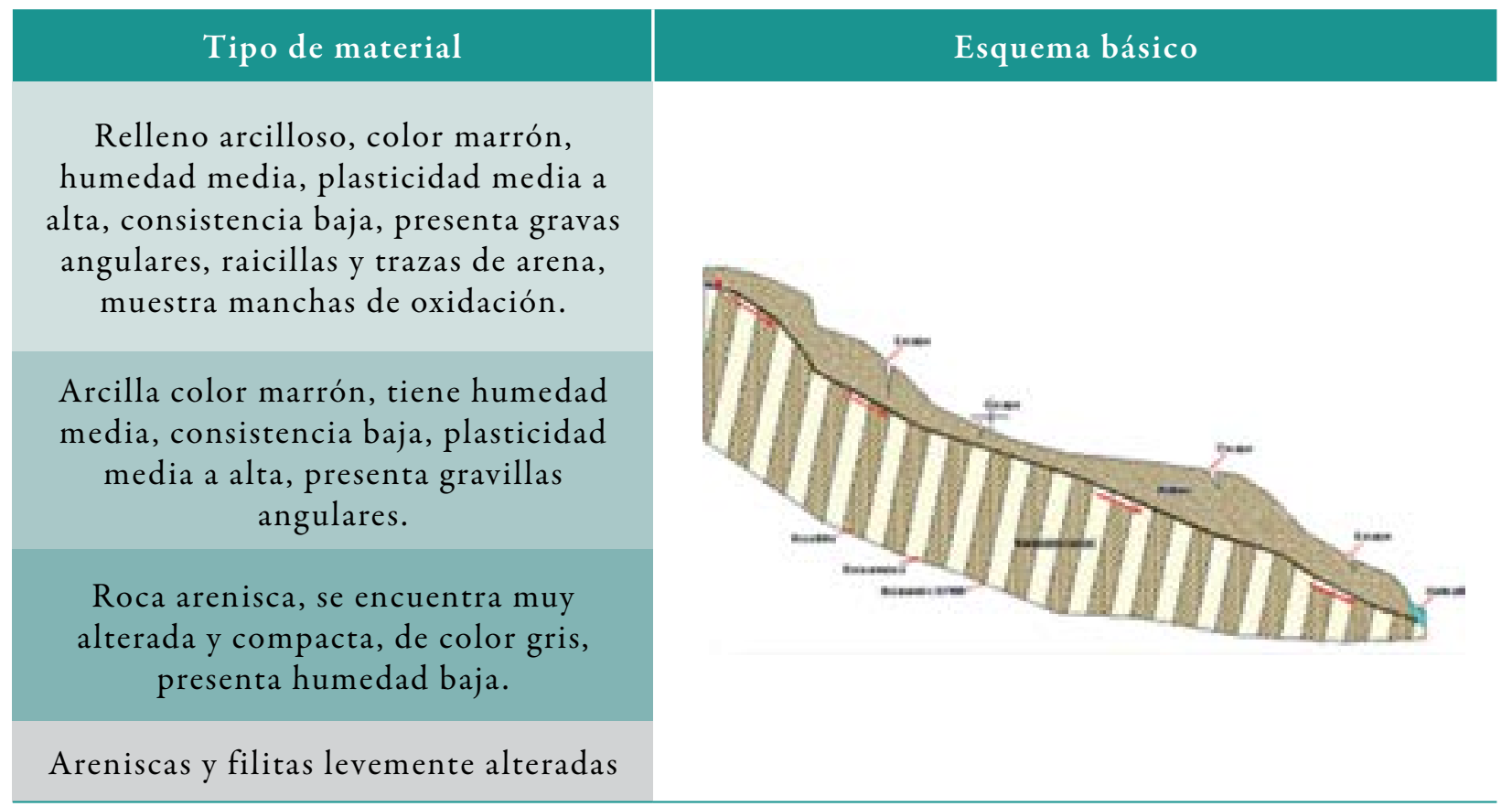

\section{Nivel freático}

A continuación (tabla 5), se muestra la relación del nivel freático con cada uno de los sondeos realizados:

Tabla 5. Niveles freáticos reportados.

\begin{tabular}{|c|c|c|c|c|c|c|c|c|c|c|c|c|c|}
\hline SONDEO & $\begin{array}{c}\text { Prof. } \\
\text { NF } \\
(\mathrm{m})\end{array}$ & SONDEO & $\begin{array}{c}\text { Prof. } \\
\text { NF } \\
(\mathrm{m})\end{array}$ & SONDEO & $\begin{array}{c}\text { Prof. } \\
\text { NF } \\
(\mathrm{m})\end{array}$ & SONDEO & $\begin{array}{l}\text { Prof. } \\
\text { NF } \\
(\mathrm{m})\end{array}$ & SONDEO & $\begin{array}{c}\text { Prof. } \\
\text { NF } \\
(\mathrm{m})\end{array}$ & SONDEO & $\begin{array}{c}\text { Prof. } \\
\text { NF } \\
(\mathrm{m})\end{array}$ & SONDEO & $\begin{array}{c}\text { Prof. } \\
\text { NF } \\
(\mathrm{m})\end{array}$ \\
\hline SM 1 & N.R & SM 11 & N.R & SM 21 & N.R & SM 31 & N.R & SM 41 & N.R & SM 51 & N.R & SM 61 & N.R \\
\hline SM 2 & 2,0 & SM 12 & N.R & SM 22 & N.R & SM 32 & N.R & SM 42 & N.R & SM 52 & N.R & SM 62 & N.R \\
\hline SM 3 & 5,5 & SM 13 & $0,8^{*}$ & SM 23 & N.R & SM 33 & N.R & SM 43 & N.R & SM 53 & N.R & SM 63 & N.R \\
\hline SM 4 & 5,5 & SM 14 & N.R & SM 24 & N.R & SM 34 & N.R & SM 44 & N.R & SM 54 & N.R & SM 64 & N.R \\
\hline SM 5 & 5,5 & SM 15 & 5,0 & SM 25 & N.R & SM 35 & N.R & SM 45 & N.R & SM 55 & N.R & SM 65 & N.R \\
\hline SM 6 & N.R & SM 16 & 0,8 & SM 26 & N.R & SM 36 & N.R & SM 46 & N.R & SM 56 & N.R & SM 66 & N.R \\
\hline SM 7 & N.R & SM 17 & N.R & SM 27 & N.R & SM 37 & N.R & SM 47 & N.R & SM 57 & N.R & SM 67 & N.R \\
\hline SM 8 & 6,5 & SM 18 & N.R & SM 28 & N.R & SM 38 & N.R & SM 48 & N.R & SM 58 & N.R & SM 68 & N.R \\
\hline SM 9 & N.R & SM 19 & 3,0 & SM 29 & N.R & SM 39 & N.R & SM 49 & N.R & SM 59 & N.R & & \\
\hline SM 10 & N.R & SM 20 & N.R & SM 30 & N.R & SM 40 & N.R & SM 50 & N.R & SM 60 & N.R & & \\
\hline
\end{tabular}


Los niveles freáticos encontrados en la exploración del subsuelo no reflejan la alta humedad que se evidencia en la zona. Lo anterior puede ser debido a la época en que se realizaron los sondeos (temporada seca) o al continuo flujo de agua hacia el estrato de arenisca fracturada que subyace a los rellenos y a los materiales blandos.

\section{Ensayos de laboratorio}

Una vez terminada la etapa de exploración del subsuelo, se ejecutaron los siguientes tipos de ensayos de laboratorio: límites de Atterberg, humedad natural, pesos unitarios y granulometrías. En la tabla 6 se presenta el número de ensayos ejecutados.

\section{Tabla 6. Número de ensayos de laboratorio desarrollados.}

\begin{tabular}{|c|c|}
\hline TIPO DE ENSAYOS & Número \\
\hline Límites de Atterberg & 68 \\
\hline Humedad natural & 68 \\
\hline Peso unitario & 68 \\
\hline Granulometría & 68 \\
\hline
\end{tabular}

\section{Condición general}

Los movimientos complejos consisten en la combinación de uno o más tipos de movimientos. Muchos deslizamientos son complejos, aunque por lo general un tipo de movimiento domina sobre los demás en ciertas áreas dentro del deslizamiento o durante cierto tiempo. Para el caso del $\mathrm{KP} 67+500$, existen dos zonas fácilmente diferenciables: la parte alta y la parte baja. La parte alta se caracteriza por tener el afloramiento de roca cerca al DDV y porque el espesor de los rellenos se incrementa a medida que se alejan, formando superficies de falla rotacionales con radios de giro grandes y en dirección casi perpendicular al DDV. La parte baja evidencia un espesor de los materiales constante y en sentido de la mayor pendiente del terreno paralela al DDV. En esta zona se evidencian indicios de movimientos traslacionales con superficies de falla dadas en el contacto entre la roca alterada y el material blando.

En la corona y en el flanco izquierdo, que se marca claramente sobre el DDV, con alturas variables entre $0,8 \mathrm{~m}$ y $1,5 \mathrm{~m}$, se pude apreciar claramente la magnitud del movimiento y las deformaciones unitarias que fueron útiles en el momento de calibrar la modelación geotécnica.

Figura 4. Esquema general del deslizamiento KP67+500. 


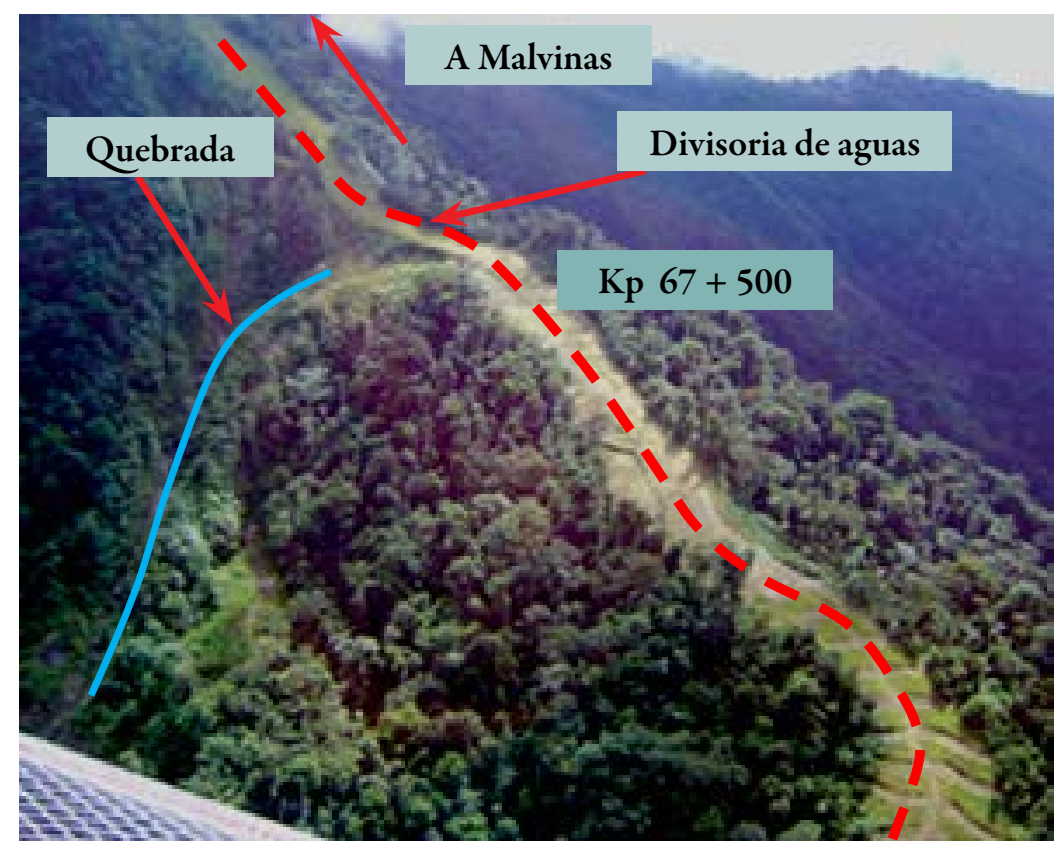

Figura 5. Panorámica del sector KP 67+500.

Plasticidad de los materiales

Según la clasificación de suelos propuesta por el Sistema Unificado de Clasificación (USCS) y los resultados de los ensayos de laboratorio, en el área de estudio predominan arcillas de baja plasticidad y arenas de grano fino de humedad baja, con una baja tasa de contenido de limo y material orgánico. En la figura 6 se presenta la carta de plasticidad de los materiales del sector.

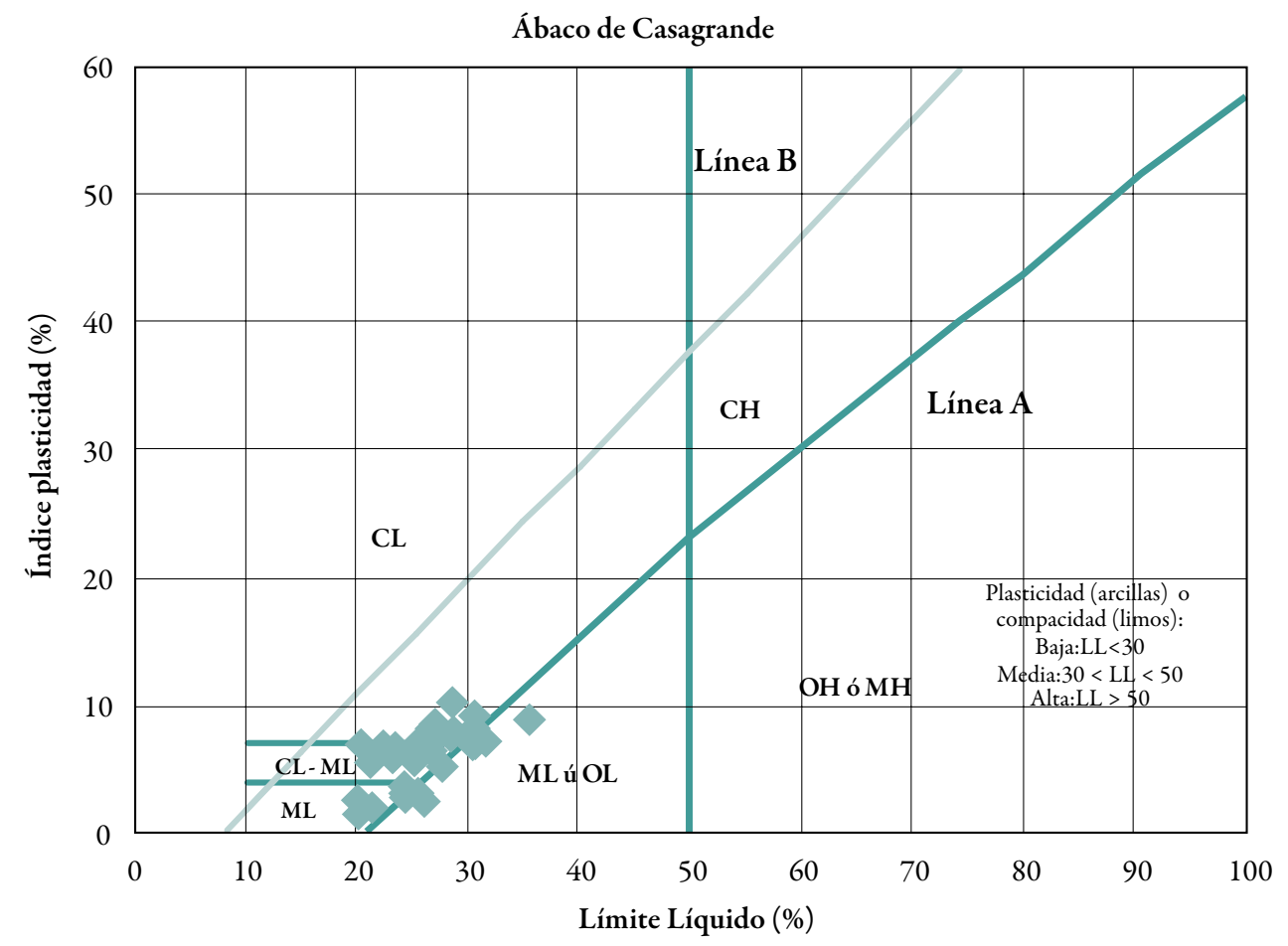

Figura 6. Carta de plasticidad de los materiales del sector. 
Tabla 7. Valores del ángulo de fricción y cohesión para los materiales identificados.

\begin{tabular}{|c|c|c|c|c|c|c|c|}
\hline \multirow{2}{*}{ MATERIAL } & \multirow{2}{*}{$\begin{array}{l}\text { P. UNITARIO } \\
\left(\text { ton } / \mathrm{m}^{3}\right)\end{array}$} & \multicolumn{3}{|c|}{ COHESIÓN (ton/m²) } & \multicolumn{3}{|c|}{ FRICCIÓN (grados) } \\
\hline & & Máx & Mín & Retrosp. & Pico & Res. & Retrosp. \\
\hline Relleno & 1,7 & 0,7 & 0 & 0,4 & 25 & 19 & 22 \\
\hline Limo, arcilla & 1,7 & 2,0 & 0 & 1,2 & 40 & 18 & 30 \\
\hline Arenisca fracturada & 1,8 & 1,0 & 0 & 0,5 & 45 & 34 & 37 \\
\hline Roca sana & 1,8 & & & & & & \\
\hline
\end{tabular}

\section{Resistencia al corte}

Teniendo en cuenta la interpretación de los resultados de laboratorio y el número de golpes del ensayo de SPT, se determinó la resistencia del suelo, y siguiendo la metodología propuesta por Álvaro González (1997), se obtuvieron los datos del ángulo de fricción pico y residual, los valores de cohesión y el peso unitario para cada uno de los materiales identificados en la exploración del subsuelo presentados en la tabla 7 .

\section{Descripción y esquemas de obras}

\section{Muro de contención con cimentación profunda}

Con el fin de proteger la tubería y aislar el movimiento hacia la parte baja del talud, se plantea la construcción de un (1) muro de gaviones con pilotaje profundo, paralelo a la tubería. La longitud total del muro principal será de $176 \mathrm{~m}$, con tres niveles de gavión $(3,0 \mathrm{~m}$ de altura) y a una distancia promedio de $6,0 \mathrm{~m}$ del ducto de NGL. La cota de cimentación del muro varía de acuerdo con la forma del terreno, entre las cotas de $1518 \mathrm{msnm}$ y $1571 \mathrm{msnm}$. A partir de estas cotas, se debe iniciar la cimentación profunda con pilotes metálicos hincados hasta llegar a un material competente compuesto por una roca arenisca color gris. El volumen total del muro a construir es de $1400 \mathrm{~m}^{3}$.

Es importante destacar que la cimentación para estos muros de gaviones será profunda, mediante el hincado y arriostramiento de un sistema de pilotes con tubería metálica de $\mathrm{D}=6$ ", cuya longitud oscilara entre $7,0 \mathrm{~m}$. y $12,0 \mathrm{~m}$ o hasta llegar al estrato competente de roca, en cada caso. El espaldón de los muros de gaviones deberá llevar un filtro con el fin de evitar un aumento de la presión hidrostática que pueda inducir el volcamiento. Dicho muro funciona de manera monolítica debido a las riostras en la parte superior del sistema de pilotaje, ya que los pilotes atraviesan los muros de gavión en sus primeras 2 hiladas.

\section{Diseño del muro de gaviones}

Se realizó el chequeo de estabilidad de los muros para las siguientes condiciones, teniendo en cuenta los valores mínimos de factores de seguridad:

- Revisión por volteo respecto a la punta del muro.

- Revisión de la falla por deslizamiento a lo largo de la base (como el muro de gavión está anclado al sistema de pilotes y trabajan de manera monolítica, se considera que esta condición se cumple).

- Revisión de la falla por capacidad de carga de la base (puesto que los pilotes sobre los cuales está empotrado el gavión deben alcanzar el nivel de roca, se asume que esta condición se cumple). 


\section{Cimentación profunda}

Sobre toda la longitud de los muros, se debe realizar un pilotaje profundo con pilotes de acero de $\mathrm{D}=6$ " arriostrados entre sí, debido a que, según el estudio de suelos, el nivel de cimentación está entre 7,0 y $12,0 \mathrm{~m}$ de profundidad.

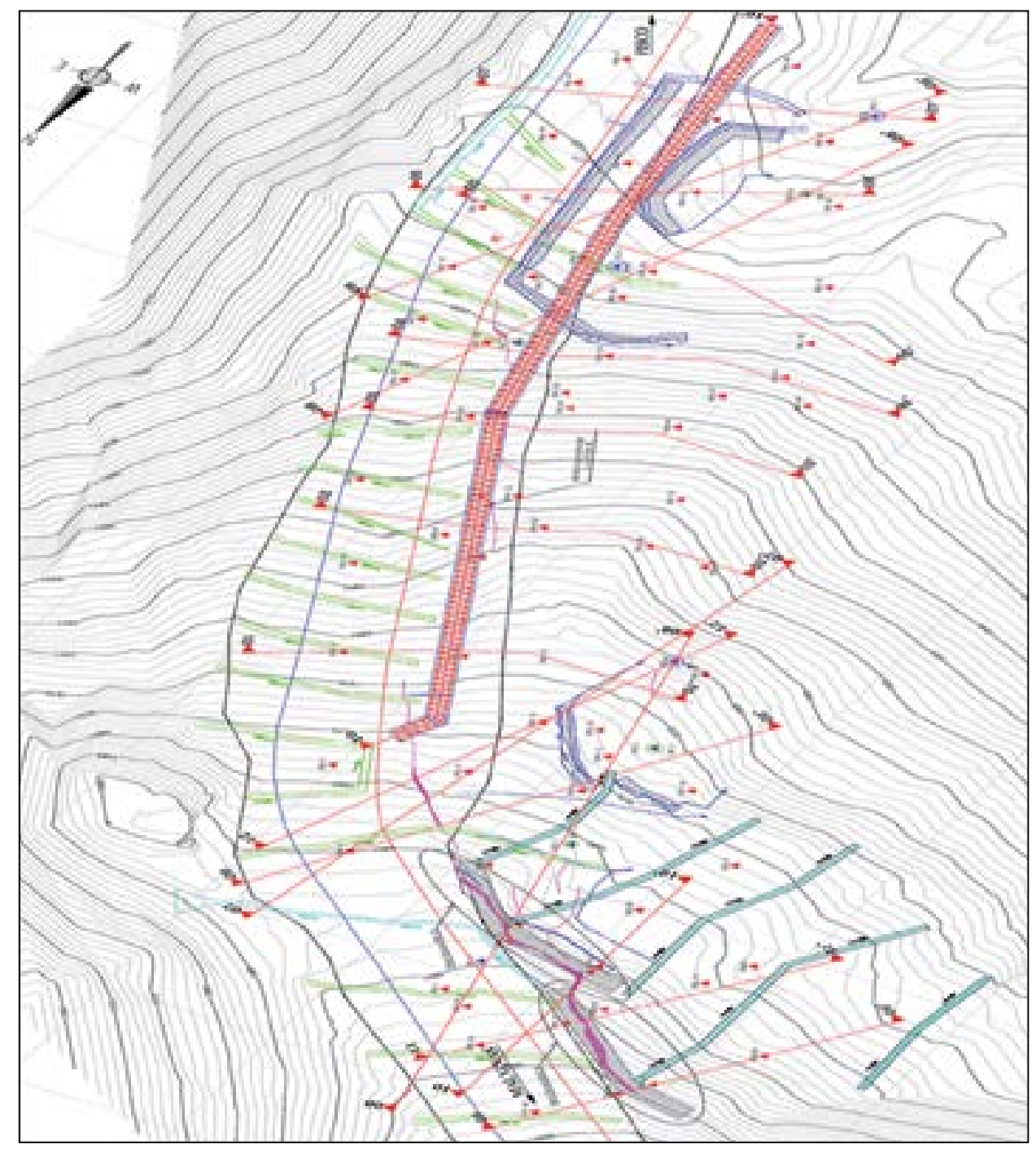

Figura 7. Obras planteadas.

\section{Excavación para el hincado de pilotes}

La excavación se realizará dejando taludes temporales no mayores de 45 grados, y una altura no mayor de 4,0 m. El material excavado deberá protegerse con polipropileno para evitar su humedecimiento ante las lluvias de la zona. El ancho de la banca será de 3,0 m, y allí deberá colocarse un sistema de filtros para la salida del agua y evitar que la zanja se sature.

\section{Hincado de pilotes}

Unavezrealizada la excavación, se procederáal hincado de pilotes de acero de $\mathrm{D}=6$ ", fuertemente arriostrados con tubería metálica de 6", soldada. En la figura 8 se detalla la geometría y disposición de los pilotes y riostras. La profundidad de hincado será variable, entre los 7,0 y 12,0 m de profundidad, a la cual se llegará mediante una excavación de $3 \mathrm{~m}$ aproximadamente. 


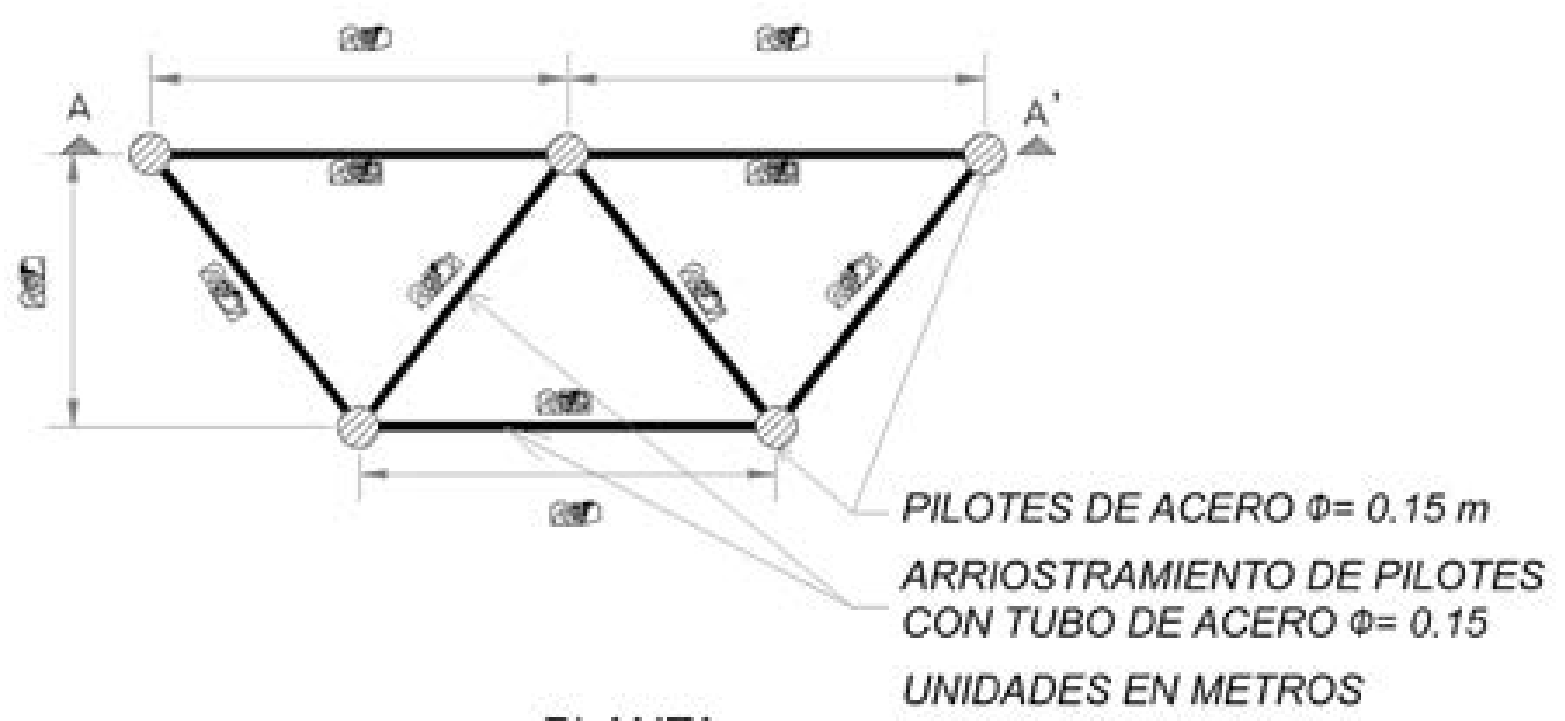

\section{PLANTA}

Figura 8. Disposición de pilotes y riostras.

De acuerdo con la longitud de los muros de gaviones, se estima que serán necesarios un total de $3130 \mathrm{~m}$ de tubería de acero de $\mathrm{D}=6$ ", representados en 522 tubos de $6 \mathrm{~m}$ de longitud.

Para este proceso se requiere una maquina piloteadora CAT312, acondicionada para este tipo de trabajo.

\section{Construcción del muro de gaviones}

Una vez finalizado el arriostramiento de los cabezales del sistema de pilotaje profundo, se procederá a la construcción de un muro de gaviones con coloide StaSoil ISO $\left(250 \mathrm{ml} / \mathrm{m}^{3}\right)$. El muro contará con $3 \mathrm{~m}$ de altura y con un traslapo del sistema de cabezales de los pilotes con el cuerpo del gavión de $2 \mathrm{~m}$. El llenado de los sacos de suelo cemento se realizará con el material excedente del corte para el hincado de los pilotes.

\section{Proceso constructivo}

A continuación se ilustran los pasos a seguir durante el proceso constructivo (figura 9).
- Desbroce y excavación hasta la cota proyectada, donde se tiene estimado que estará el primer nivel de gaviones.

- Hincado de los pilotes de $\varnothing=6$ " para la cimentación profunda, de acuerdo con las especificaciones, distribución y diseños en planos.

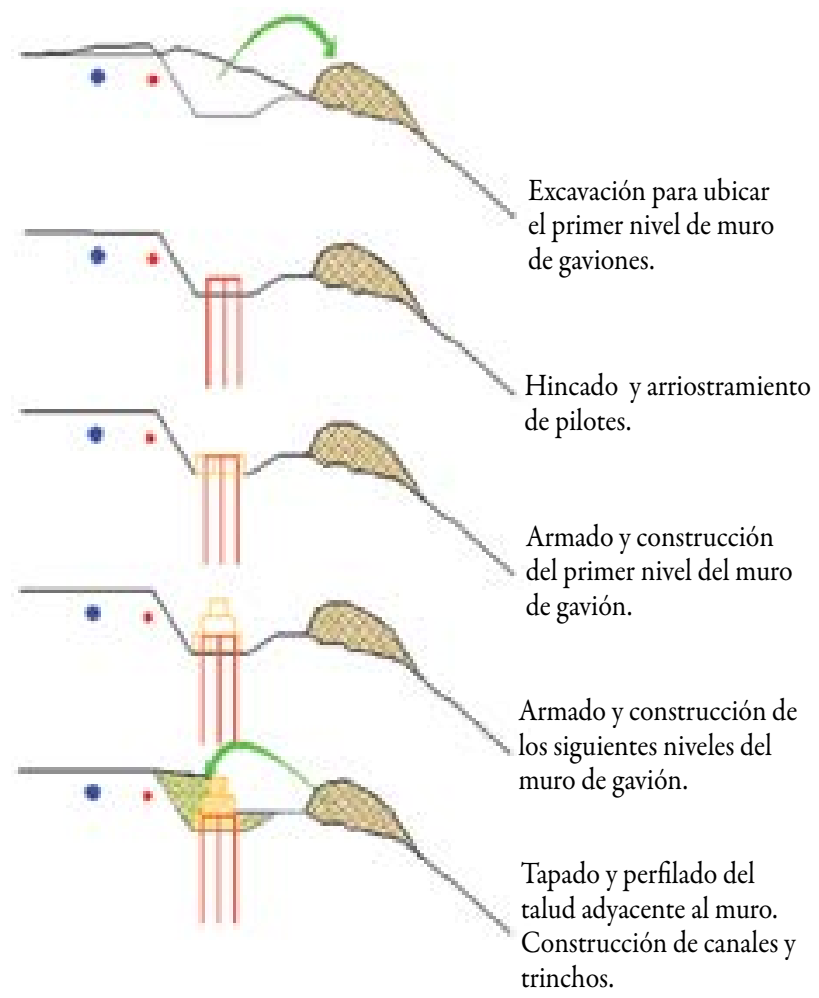

Figura 9. Esquema básico del proceso constructivo. 
- Arriostramiento de los pilotes con tubos de $\varnothing=6 "$

- Armado y construcción de gaviones de cimentación.

- Construcción de los niveles subsiguientes del muro de gaviones con relleno en el trasdós.

\section{Construcción de canal colector}

Se construirá un canal colector en la margen izquierda del DDV, con una longitud de 60 $\mathrm{m}$. El canal se revestirá con sacos con suelo cemento en una relación de 6:1. El ancho del canal será de $40 \mathrm{~cm}$ e irá incrementándose 10 $\mathrm{cm}$ cada $30 \mathrm{~m}$ de longitud. La altura del canal será de $0,4 \mathrm{~m}$ y se incrementará hasta llegar a $0,6 \mathrm{~m}$, en el descole final (ver figuras 10 y 11 ). A continuación se indican las especificaciones que se deben cumplir.

\section{Cálculo de caudales máximos esperados}

Para el cálculo de los caudales máximos esperados, se utilizó la fórmula racional. Este planteamiento se identifica con la ecuación:

$$
Q=k i A c
$$

donde $Q$ es el caudal pico de la creciente $\left(\mathrm{m}^{3} / \mathrm{s}\right)$, $k$ es un factor de conversión que en el sistema métrico es igual a $0,00278\left(\mathrm{~m}^{3} / \mathrm{s} / \mathrm{ha} \mathrm{mm} / \mathrm{h}\right)$, $i$ es la intensidad máxima para la frecuencia de diseño (con una duración igual al tiempo de concentración de la cuenca vertiente $(\mathrm{mm} / \mathrm{h}), A$ es el área de la cuenca (ha), y $c$ es el coeficiente de escorrentía, con valor numérico entre $0 \mathrm{y}$ 1, el mismo que depende de las características fisiográficas de la cuenca y de la cobertura del suelo.
Las variables de trabajo fueron las siguientes:

- Área de drenaje: 2,5 ha.

- Coeficiente de escorrentía.

El coeficiente de escorrentía es la relación entre la tasa pico de escorrentía directa y la intensidad promedio de la precipitación en una tormenta. Debido a la alta variabilidad de la intensidad de la precipitación, este valor es de difícil determinación (máxime si se emplea la información). Por tal motivo, se optó por estimar el coeficiente de escorrentía teniendo en cuenta el uso actual del suelo. En concordancia con esto, se asignó un coeficiente de escorrentía de 0,55, según la CDMB.

- Tiempo de concentración

El tiempo de concentración, o tiempo requerido para que el agua caída en el punto más alejado de la cuenca hidrográfica fluya a una sección específica de análisis en el drenaje, está en función de las características topográficas de la cuenca. En este caso, por tratarse de microcuencas de pendiente media a alta, el tiempo de concentración puede evaluarse gracias a la formula de Kirpich:

$$
T_{c}=4 \times\left(\frac{L}{\sqrt{S}}\right)^{0,77}
$$

Donde:

$\mathrm{T}_{\mathrm{c}}=$ tiempo de concentración ( $\mathrm{min}$ )

$\mathrm{L}=\operatorname{Longitud}(\mathrm{km})$

$\mathrm{S}=$ Pendiente $(\mathrm{m} / \mathrm{m})$

- Caudales máximos

Con los valores anteriores, se calculan los caudales máximos para tres diferentes períodos de retorno: 5, 10 y 25 años. Estos resultados se presentan en la tabla siguiente: 
Tabla 8. Caudales máximos.

\begin{tabular}{c|c|c|c}
$\begin{array}{c}\text { “TC" TIEMPO DE } \\
\text { CONCENTRACIÓN } \\
\text { ADOPTADO }(\mathrm{min})\end{array}$ & $\begin{array}{c}\text { TIEMPO DE } \\
\text { RETORNO }\end{array}$ & $\begin{array}{c}\text { "I" INTENSIDAD } \\
\text { DE LA LLUVIA } \\
(\mathrm{mm} / \mathrm{hr})\end{array}$ & $\begin{array}{c}\text { "Q CAUDAL } \\
\left(\mathrm{m}^{3} / \mathrm{s}\right)\end{array}$ \\
6,27 & 5 & 100 & 0,382 \\
6,27 & 10 & 150 & 0,573 \\
6,27 & 25 & 200 & 0,764 \\
\hline
\end{tabular}

De acuerdo con Jaime Suárez (1992), para el diseño de obras en el cauce de una corriente permanente se recomienda trabajar con períodos de retorno de veinticinco años, y para cunetas y taludes y en áreas de corrientes ocasionales, se puede utilizar un período de retorno de diez años.

\section{Diseño del canal colector}

Con el fin de realizar el control del agua analizada anteriormente, se debe diseñar una cuneta que logre evacuar en forma efectiva y controlada el flujo de agua en la zona. Para ello, se plantea diseñar tres cunetas: una que atraviese longitudinalmente la zona, y dos que, a una altura adecuada de recolección de aguas, se conecten después a la primera en forma transversal. Teniendo en cuenta este concepto, se detalla a continuación el análisis de diseño de las obras de drenaje.
Para el diseño del canal se utiliza el método de Manning, expresado en la fórmula:

$$
Q=\frac{A^{*} R^{\frac{2}{3}} * S^{0.5}}{n}
$$

Donde:

$A$ Área de la sección mojada

$R$ Radio hidráulico

$S$ Pendiente (calculada con base en el levantamiento topográfico)

$n$ Rugosidad

Considerando que el flujo es uniforme, la Rugosidad de Manning ( $\mathrm{n}$ ) = 0,011 para la cuneta de concreto (tomado de la CDMB).

Según lo anterior, con el caudal a movilizar y planteando el diseño de la cuneta en forma rectangular, se obtiene el equilibrio con un $\mathrm{FS}=3,75$. Los valores se muestran en la tabla siguiente:

\section{Tabla 9. Características del canal.}

\begin{tabular}{|c|c|c|c|}
\hline Variable & Valor & Unidad & Observación \\
\hline Altura & 0,40 & $\mathrm{~m}$ & Base \\
\hline Ancho & 0,40 & $\mathrm{~m}$ & \\
Espesor & 0,00 & $\mathrm{~m}$ & Rugosidad de Manning \\
Área & 0,16 & $\mathrm{~m}^{2}$ & \\
n & 0,01 & - & Pendiente de la cuneta con el terreno \\
\hline Ángulo & 35.00 & ${ }^{\circ}(\mathrm{Grados})$ & \\
\hline Pendiente & 0,70 & $\mathrm{~m} / \mathrm{m}$ & Pendiente de la cuneta con el terreno \\
\hline Perímetro mojado & 1,20 & $\mathrm{~m}$ & \\
Radio hidráulico & 0,13 & $\mathrm{~m}$ & Caudal de diseño. Lluvia crítica \\
\hline Q(diseño) & 763,89 & $1 / \mathrm{s}$ & Factor de distribución \\
\hline FD & 1,00 & - & Caudal de diseño final \\
\hline Q(final) & 763,89 & $1 / \mathrm{s}$ & \\
\hline Q manning & 3,18 & $\mathrm{~m}^{3} / \mathrm{s}$ & Factor de seguridad \\
\hline Q manning & 2541,30 & $1 \mathrm{itros} / \mathrm{s}$ & \\
\hline FS & 3,33 & - & \\
\hline
\end{tabular}



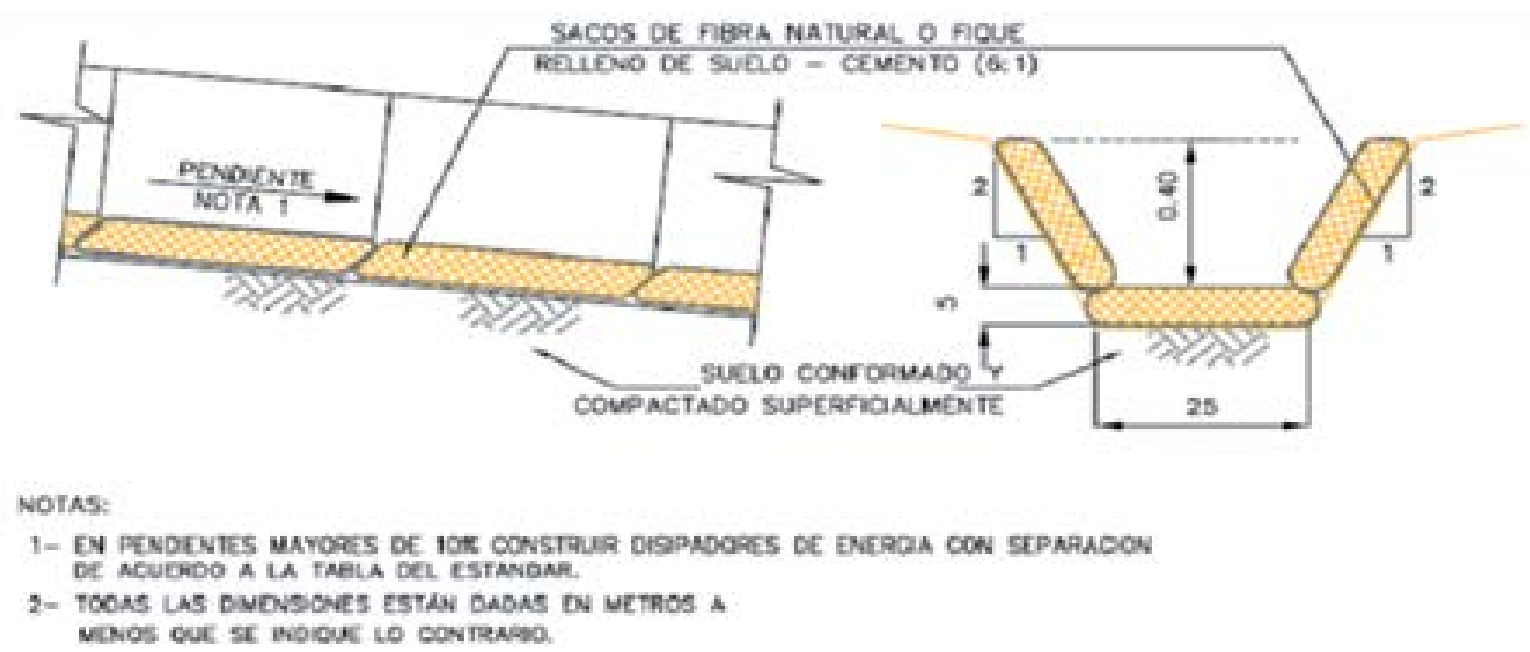

Figura 10. Detalle del canal colector.

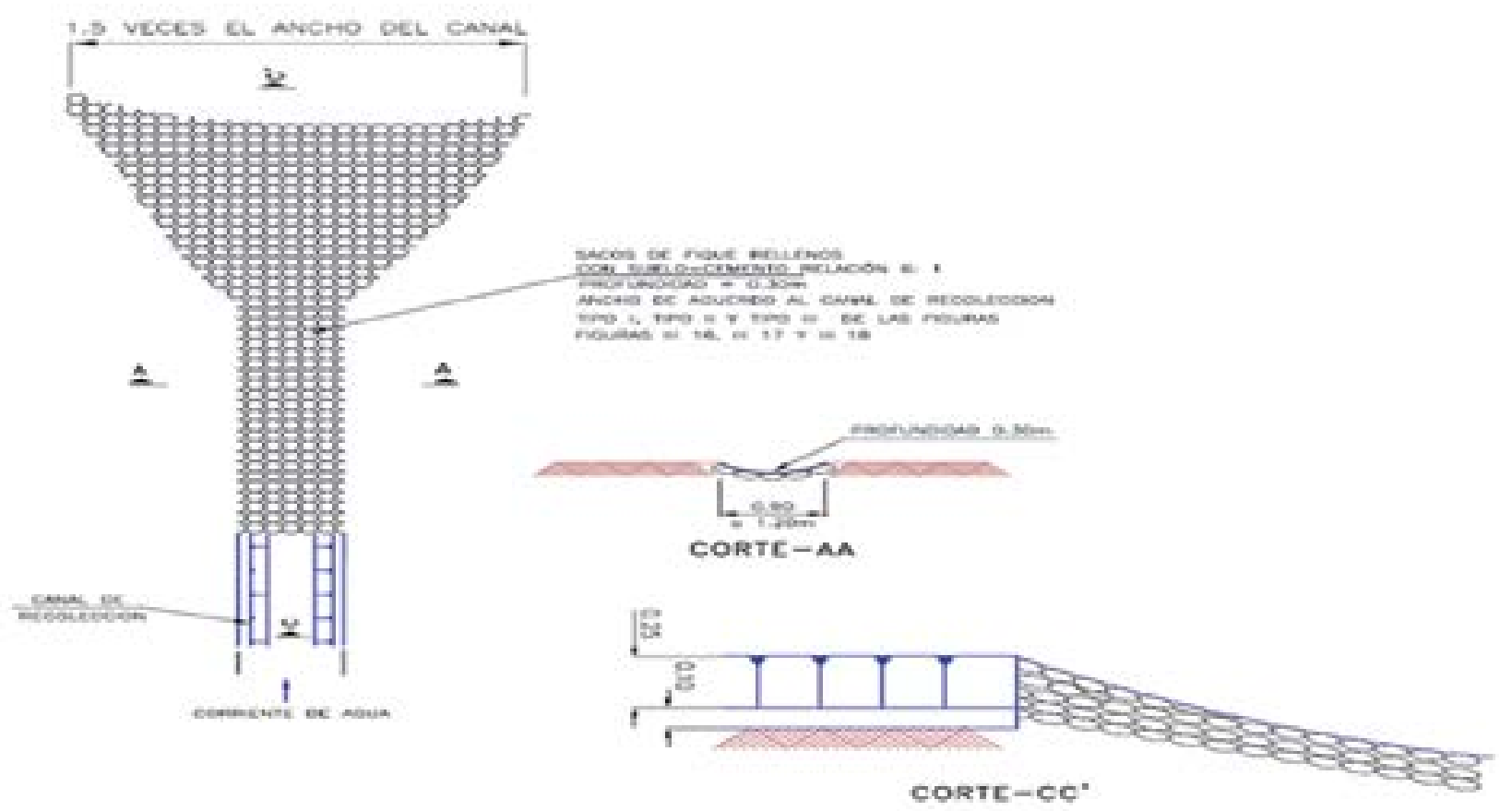

Figura 11. Detalle del descole.

Manejo superficial de aguas

Para mantener un adecuado manejo de aguas, descargando sobre el costado izquierdo (sentido del flujo) del DDV, se deben reconstruir los cortacorrientes (en sacos de suelo cemento y con relación de 6:1) con descoles, de acuerdo con las especificaciones y distribución que se localiza en los planos de diseño. En total se planea construir $300 \mathrm{~m}$ de cortacorrientes.
Muro de contención (acopio en la parte alta del DDV)

Debido a que en la parte alta del DDV se conformó un acopio durante la etapa de construcción, el cual no fue estabilizado adecuadamente, se presenta un movimiento de este, generando un proceso retrogresivo que puede afectar a futuro el DDV. Es preciso resaltar que este es un movimiento diferente que busca 
aislar a los ductos. Por tal motivo, se construirán dos muros de gavión en saco con suelo coloide en la parte alta del DDV, con la finalidad de aislar este proceso de movimiento de la zona de los ductos. Estos muros se cimentarán sobre el estrato competente (roca). Los muros tendrán 36 y $34 \mathrm{~m}$ respectivamente, con tres niveles, y se instalará un filtro geodren en el trasdós para disipar las presiones hidrostáticas. Este tendrá un volumen total $470 \mathrm{~m}^{3}$. Adicionalmente, se debe construir un sistema de trincheras drenantes "abiertas" en la zona del acopio (ver figura 12), a no menos de $2 \mathrm{~m}$ de profundidad, con la finalidad de reducir las ratas de infiltración de agua y así disminuir el movimiento que se genera en esta zona. Se construirán $201 \mathrm{~m}$ de trinchera.

\section{CONCLUSIONES}

- Conforme a lo observado tanto en la exploración del subsuelo como durante la visita de campo, y atendiendo al comportamiento del modelo geotécnico debido al tipo de material y pendiente del terreno que se encuentra sobre el costado derecho del DDV, es importante resaltar que con el diseño y construcción de las obras se logra el aislamiento del ducto de este deslizamiento pero no se estabiliza el movimiento ladera abajo.

- Para evitar la saturación de la zona de trabajos, se debió cubrir el área con plástico y malla arpillera durante las noches, en los

\section{DETALLE DE TRINCHERA DRENANTE}

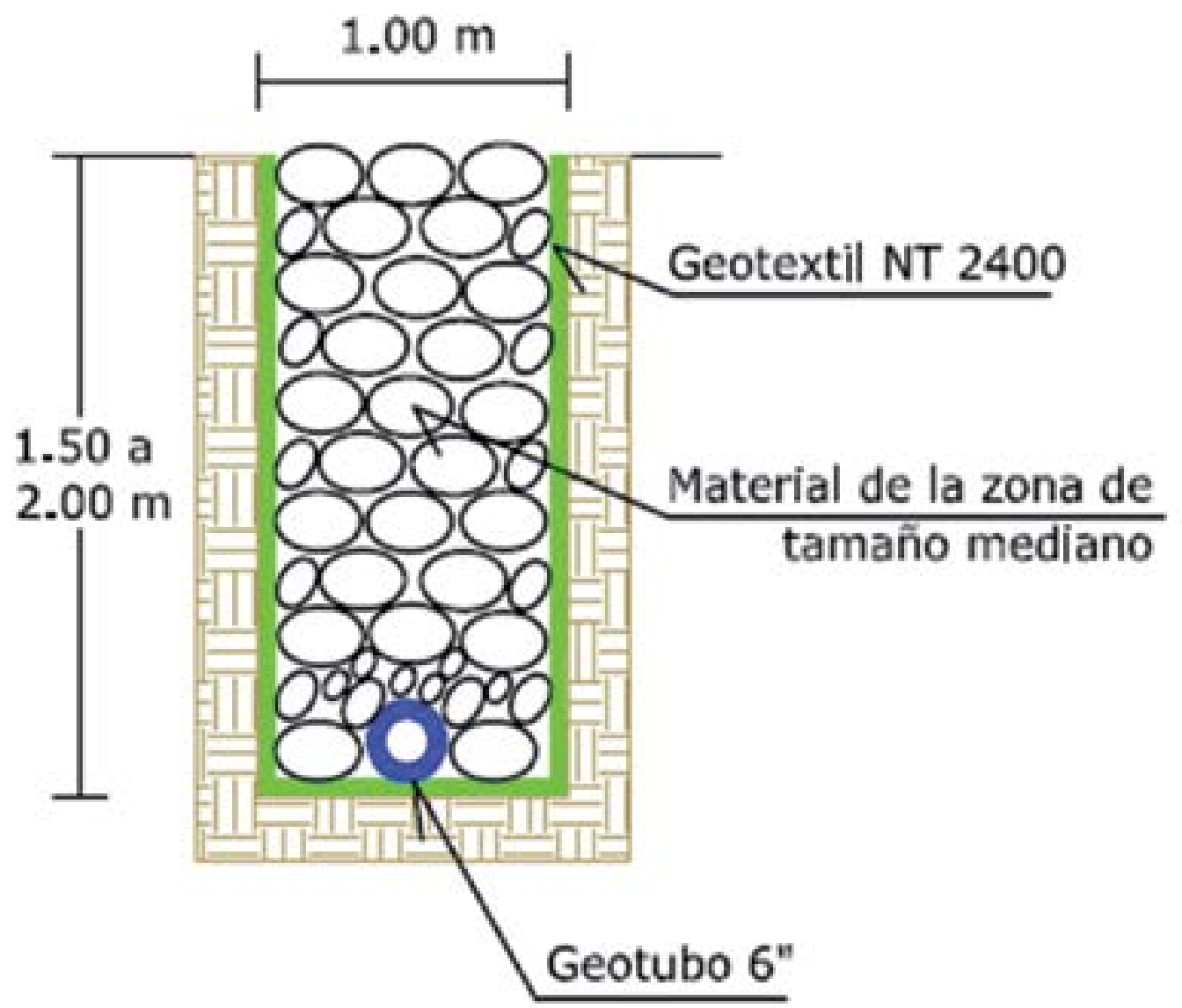

Figura 12. Sección típica de las trincheras drenantes. 
momentos de lluvia y durante el tiempo en el que no se estaban ejecutando los trabajos.

- Antes del inicio de labores, se contó con el visto bueno de RRCC, que comunicaba al posesionario, autoridades locales y comunidades acerca de las labores a realizar en la zona de trabajo, con la finalidad de evitar conflictos de tipo social.

- En el área de la obra predominan arcillas de baja plasticidad y arenas de grano fino de humedad baja, con una baja tasa de contenido de limo y material orgánico.

- El deslizamiento de la zona en estudio es complejo, ya que involucra superficies de falla rotacionales de gran radio con tendencia traslacional y direcciones de movimientos no homogéneas producidos en diferentes etapas

- El tipo de movimiento en el área es complejo, pues presenta superficies de falla rotacionales de gran radio y bloques con movimiento traslacional en la zona de contacto entre la roca y el material blando.

\section{RECOMENDACIONES}

- Una vez finalizadas las tareas geotécnicas, de limpieza, reconformación y siembra del DDV, se deben realizar monitoreos e inspecciones periódicas del sector intervenido.

- Una vez finalizada la ejecución de todos los trabajos, se hace necesaria la instalación de la instrumentación geotécnica (inclinómetros, piezómetros y mojones topográficos) para poder iniciar el monitoreo del comportamiento de las obras construidas en la escala de tiempo.

- Debe cumplirse con los procedimientos de medio ambiente y seguridad industrial durante la ejecución de los trabajos, además de asegurarse de que al final las áreas queden libres de basura u otros materiales.

- Debe cumplirse con los procedimientos de trabajos en cimentaciones y trabajos en espacios confinados, que incluyen trabajos en zonas con taludes, movimiento de material por excavación, soportes de taludes sobre los costados de la zona de trabajo y demás que impliquen la ejecución de este plan de trabajo.

- Se debe garantizar en campo que los pilotes sean hincados hasta la profundidad de diseño o hasta alcanzar la roca arenisca identificada en los sondeos profundos. De no ser así, se deben detener las labores y verificar otra alternativa. De ser necesario se deberá conseguir una máquina más potente, de modo que se alcance el fin propuesto. De lo contrario, la obra no cumplirá su objetivo.

\section{GLOSARIO}

ANP: Área Natural Protegida

BID: Banco Interamericano de Desarrollo.

COGA: Compañía Operadora del Gas del Amazonas.

CND: Consejo Nacional Descentralizado de Camisea.

CCNN: Comunidades nativas.

CV: Cobertura vegetal.

DDV: Derecho de vía.

DIGESA: Dirección General de Salud Ambiental.

DIA: Declaración de impacto ambiental.

DIRESA: Dirección Regional de Salud.

EIA: Estudio de impacto ambiental.

EPPs: Equipos de protección personal. 
EPS: Empresa prestadora de servicios.

GDP: Gobierno del Perú.

GLP: Gas licuado de petróleo.

LGN: Gas natural licuado.

MEF: Ministerio de Economía y Finanzas.

MINAM: Ministerio del Medio Ambiente.

MINEM: Ministerio Nacional de Energía y Minas.

MINSA: Ministerio de Salud.

OSINERGMIN: Organismo Supervisor de la Inversión en Energía y Minería.

PDT: Plan de trabajo.

PDL: Plan de desarrollo local.

RRCC: Relaciones comunitarias.

RRSS: Residuos sólidos.

STD: Sistema de transporte por ductos.

\section{REFERENCIAS BIBLIOGRÁFICAS}

- Compañía Operadora del Gas del Amazonas (COGA), 2009. Especificaciones técnicas de obras geotécnicas. Lima.

- Compañía Operadora del Gas del Amazonas (COGA), 2008-2009. Informes Técnicos de la Oficina Técnica y Geotecnia. Lima.

- Instituto Nacional de Vías, 1998. Manual de estabilidad de taludes. Bogotá, Colombia.

- Ingeniería y Geotecnia Ltda., septiembre de 2007. Taller sobre evaluación de riesgos por geoamenazas para el gasoducto de Camisea. Kiteni, Perú.

- Jaime Suárez, 2009. Deslizamientos. Tomo I, capítulo 5. Bucaramanga, Colombia,

- Jaime Suárez, 1998. Deslizamientos y estabilidad de taludes en zonas tropicales. Bucaramanga, Colombia, 